\title{
Using Trace Element Ratios to Establish Correlations in Brick and Terra Cotta
}

\author{
Emily Steiner ${ }^{1}$, Pippa Merrick ${ }^{2}$, Susan Tunick ${ }^{3}$, Kathryn Tierney ${ }^{4}$, Hyojin Lee $^{1}$, and William Carty ${ }^{1}$ \\ ${ }^{1}$ New York State College of Ceramics at Alfred University, Alfred, NY 14802 \\ ${ }^{2}$ Historical Hillsborough Research Group, Hillsborough, NC \\ ${ }^{3}$ Friends of Terra Cotta, New York, NY \\ ${ }^{4}$ Boston Valley Terra Cotta, Orchard Park, NY
}

Determining the provenance of ceramic materials, such as brick or terra cotta, but also potentially porcelain and other fired products, is of interest to historical societies and conservation groups. One approach has been to evaluate color or mineralogy, but these properties can be influenced by the firing temperature and atmospheric conditions (i.e., degree of oxidation or reduction) in the kiln. Analysis conducted to identify the source rock for clay deposits in Georgia proposed the use of trace element level ratios, as this measure would be insensitive to weathering [1]. Specifically, it is not the level of the trace elements, but the ratio of selected trace elements that would remain constant. The level of the trace element increases with heat treatment due to the loss of volatiles from the body during firing.

Three production bodies from Boston Valley Terra Cotta (BVTC) were tested in the green (unfired) and fired state to: (1) form a baseline, (2) identify candidate trace elements, (3) demonstrate that firing does not change the ratio of trace elements, and (4) to establish the range of error associated with the measurements. As expected, the trace element levels increases slightly with firing but the ratio of the trace elements remained similar.

To determine which elements provided the most reliable candidates, ICP-ES analysis was conducted to obtain the chemistry, including trace elements (44 elements in total), on pulp samples [2]. (LF-200 Total Whole Rock Characterization, Bureau Veritas Commodities Canada Ltd., Vancouver, BC). From the chemical analysis results, elements were eliminated based on three initial criteria: (1) detection resolution (average measured level / detection level $\leq 100$ ); solubility in water; and the potential for isomorphism (such as $\mathrm{Hf}$ in $\mathrm{ZrO}_{2}$ ). This process resulted in 8 elemental candidates. The ratios of these eight were plotted to determine if unique relationships could be discerned, i.e., the elimination of overlap between elemental ratios of the three bodies. From this list four elements were identified: $\mathrm{La}, \mathrm{Ce}, \mathrm{Sm}$ and $\mathrm{Gd}$. The ratios of these four (i.e., La/Sm; Ce/Gd; etc.) are plotted in Figure 1 for the BVTC standards with measurement reliability in the form of two standard deviations about the mean.

Similar analysis was conducted on a variety of brick and terra cotta samples, as shown in Figures $2 \& 3$. The brick samples also included clay obtained from a pit proposed to be used to manufacture brick in the town in the late 1700s. Four of the clay samples grouped tightly with one outlier. The brick samples grouped well for 8 of the 11 samples, with a broader standard deviation, with three outliers. The closeness of the clay and brick samples provides a reasonable probability that these bricks came from the identified clay deposit. Additional clay and brick samples are being tested. Similar results were obtained from the terra cotta samples. These samples were obtained from 1850 s era terra cotta installations and the goal was to identify is the manufacturers were similar. Of the 11 samples tested, 8 grouped tightly and three are clearly outliers. Overall, this approach has the potential to facilitate determining provenance, providing the opportunity to establish a probability of similarity of specimens obtained from the field. 


\section{References:}

[1] T. Dombrowski, The Use of Trace Elements to Determine Provenance Relations Among Different Types of Georgia Kaolins, Ph.D. Dissertation, Indiana University, 1992.

[2] LF-200 Total Whole Rock Characterization, Bureau Veritas Commodities Canada Ltd., Vancouver, $\mathrm{BC}$.

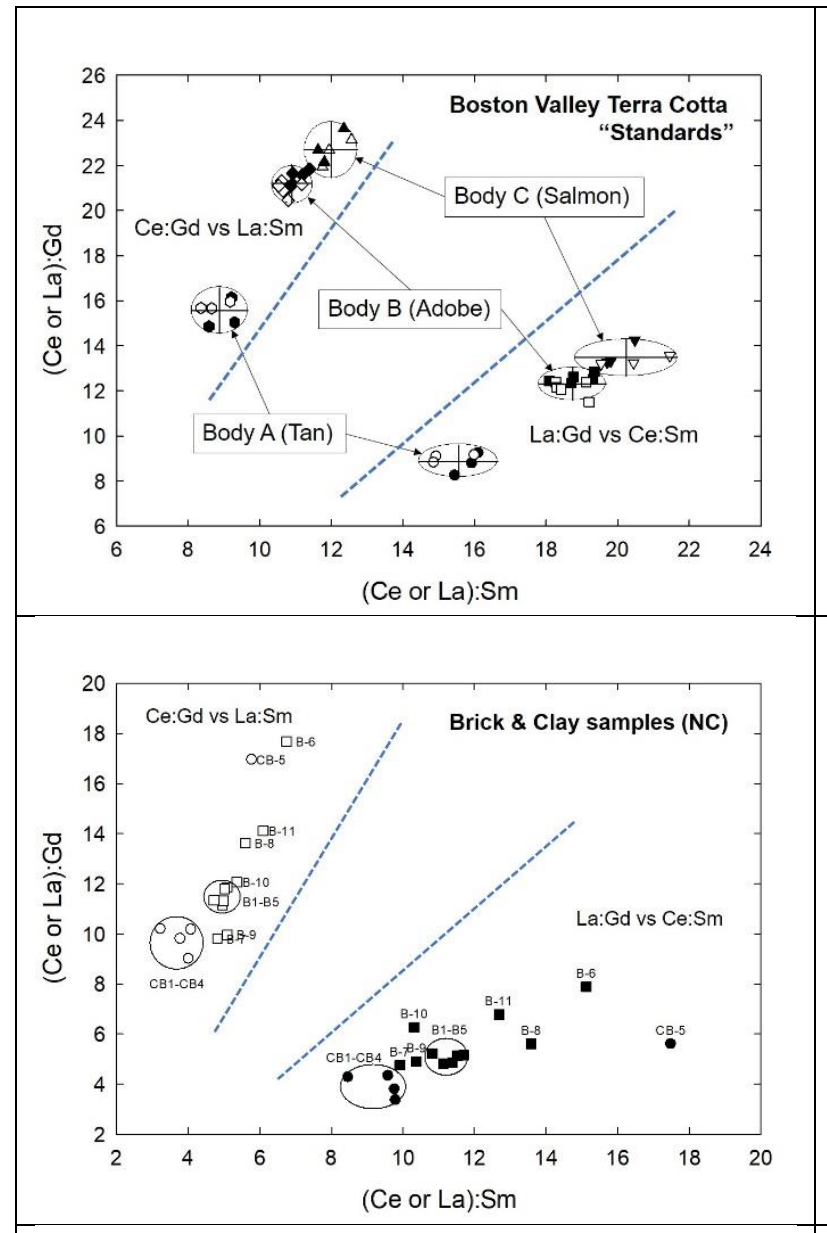

Figure 1. Elemental ratios of $\mathrm{La}, \mathrm{Ce}, \mathrm{Sm}$, and $\mathrm{Gd}$ for unfired (filled symbols) and fired (open symbols) of terra cotta clay provided by Boston Valley Terra Cotta. Three bodies were tested (with designated body color). The ovals indicate the range of two standard deviations about the mean values (and statistical analysis confirmed a normal distribution). Ce:Gd is plotted against La:Sm in the upper left and La:Gd is plotted against $\mathrm{Ce}: \mathrm{Sm}$ in the lower right.

Figure 2. Elemental ratios for clay (CB-1 thru CB-5) and brick (B-1 thru B-11) showing clear grouping of clays CB-1 thru CB-4 (outlier: CB-5) and potentially one to three groupings of brick specimens: (\#1) B-1 to B-5 \& B-10; (\#2) B-7 \& B-9; and (\#3) B-8 \& B-11 when viewing the Ce:Gd vs. La:Sm plot. When evaluated against $\mathrm{La}: \mathrm{Gd}$ vs. Ce:Sm, however, it appears that B-1 to B-5 (\#1) and \#2 (B-7 and B-9) are still valid, but the remainder appear to be discrete samples (B-6, B-8, B-10, and B-11). (Additional work is on-going to evaluate the correlation of the clay specimens with the brick specimens.)

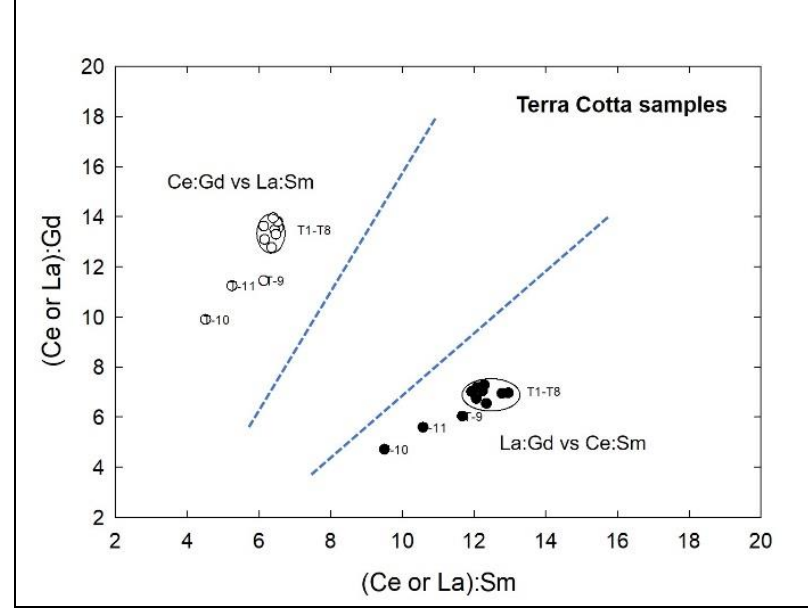

Figure 3. Elemental ratios for Terra Cotta samples extracted from several locations. The groupings of the ratios indicate that specimens $\mathrm{T}-1$ through $\mathrm{T}-8$ were likely to have been produced by the same manufacturer. Specimens T-9, T-10, and T-11 were not only highly unlikely to have been produced by the same manufacturer as T-1 through T-8 but were also likely manufactured by three separate producers. 